Objectives It is estimated that 50\% of patients diagnosed with cervical cancer never had any screening. We aimed to determine the changes in cervical cancer screening in the United States.

Methods Pap smear rates were evaluated using the Behavioral Risk Factor Surveillance System (BRFSS). SEER*Stat 8.3.8 and Joinpoint regression program 4.8.0.1 were used to calculate incidence trends.

Results In 2016, 6.28\% women in the U.S were never screened for cervical cancer. Based on race, $21.3 \%$ of Asian, 11.63\% Hispanic, $8.09 \%$ Black and $5.1 \%$ of White women have never undergone screening. The age groups with higher never screened rates were the 80 and older age cohort at $11.14 \%$ followed by the $25-29$ group at $8.87 \%$. Over the last 6 years of our study, there has been an increase of $7.4 \%$ annually of never screened rates $(p=0.008)$. In regards to age, there has been an increase in never pap was the 25-29 age group (AAPC +7.31\%, p<0.001). White and Black women have increasing never pap smear rates at $1.49 \% \quad(p=0.008)$ and $4.05 \% \quad(p<0.001)$, respectively, while Hispanic women have no change. The intersectionality of age and race shows that Black women ages 25-29 have the highest increased rate of no screening, 9.84\% annually $(\mathrm{p}<0.001)$.

Conclusions Based on this large survey, nearly one fourth of Asian women were never screened for cervical cancer in the U.S. There is also an increasing proportion of never-screened particularly in younger Black women. Further research is warranted to understand the change in screening practices in relation to vaccination and access to care.

\section{EPV045/\#220 DEVELOPMENT OF A CIRCULATING TUMOR HPV ASSAY FOR THE DETECTION OF RECURRENT CERVICAL CANCER}

R Zhang * , A Kapur, M Patankar, L Barroilhet. University of Wisconsin Hospitals and Clinics, Obstetrics and Gynecology, Madison, USA

\subsection{6/ijgc-2021-IGCS.113}

Objectives Circulating tumor DNA assays have the potential to facilitate early detection of cancer recurrence as has been demonstrated in breast, bladder, colorectal, and most recently HPV-associated oropharyngeal cancer. We sought to develop a droplet digital PCR (ddPCR) assay for the quantification of circulating tumor HPV DNA in cervical cancer patients.

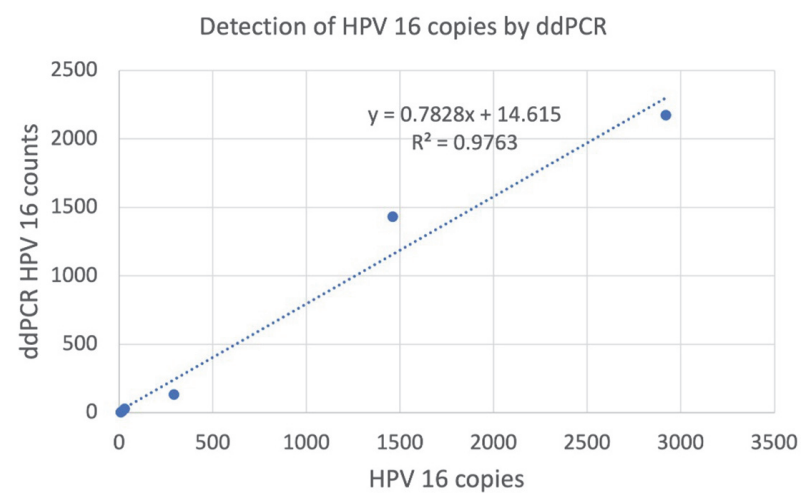

Abstract EPV045/\#220 Figure 1 Detection of HPV 16 copies by ddPCR
Methods Primers were designed to specifically detect an amplicon within the E7 gene encoded by high-risk HPV 16 and 18. Each reaction assay contained $2 \mathrm{x}$ ddPCR EvaGreen Supermix $(10 \mu \mathrm{L})$, respective primers $(4 \mu \mathrm{L})$, target DNA (1 $\mu \mathrm{L})$, and DNAase free water $(5 \mu \mathrm{L})$. Optimal annealing temperature and primer concentration were determined by running temperature and concentration titrations of PBS spiked with target gene fragments of HPV 16. Primers targeting the E7 gene of HPV 16 and 18 were combined into a single assay and HPV DNA quantification performed in control plasma samples.

Results Titration analysis demonstrated good correlation between expected HPV DNA copies and detected copies by ddPCR $\left(\mathrm{R}^{2}=0.9763\right)$. The HPV 16 and 18 assays tested individually and in combination were specific for the HPV strain of interest with no cross-reactivity to the other HPV strain.

Conclusions We developed a highly sensitive and specific ddPCR assay to detect the two dominant high-risk HPV subtypes responsible for to cervical cancer. We plan to perform a prospective pilot study to validate our assay and its clinical utility in detecting minimal residual disease and treatment response.

\section{EPV046/\#229 A REVIEW OF CERVICAL CANCER DIAGNOSED IN WOMEN OVER THE AGE OF 65}

${ }^{1} S$ Baron*, ${ }^{2}$ S Kaushik. 'University Hospitals Sussex, Obstetrics and Gynaecology, Brighton, UK; ${ }^{2}$ University Hospitals Sussex, Gynaecological Oncology, Brighton, UK

\subsection{6/ijgc-2021-IGCS.114}

Objectives To explore the incidence of cervix cancer following cessation of the UK cervical screening programme.

Methods 179 cases of cervical cancer diagnosed between 2016 and 2020 at University Hospitals Sussex were retrospectively reviewed. The screening history, grade, histology and stage of cancer were recorded.

Results Over a five-year period, 80 cases of cervical cancer were identified in the screened population. Of these $59(74 \%)$ were under 65 years and $21(26 \%)$ were over 65 years of age. An initial peak incidence was seen at 30-35 year age range, declining with further screening. Following cessation of screening, a secondary peak at $80-85$ years was noted. of those diagnosed during screening (59.3\%) were FIGO IA1 to IB2, however, only $9.5 \%$ of the over $65 \mathrm{~s}$ were early stage. Similarly, $32.2 \%$ of those within screening age presented with a grade 1 cancer, with only $4.8 \%$ over 65 years being low grade. Histology in the under 65 s revealed $44.1 \%$ were squamous cell carcinoma and $45.8 \%$ were HPV-related adenocarcinoma. In the over $65 \mathrm{~s}$ this was $76.2 \%$ and $14.3 \%$ respectively.

Conclusions Despite adherence to the screening programme, $25 \%$ of cervix cancer was diagnosed beyond screening age, approximately 16 years later. These patients were of more advanced stage and higher grade. This preliminary exploration informs the need for a wider review of cervix cancer after the age of 65 and consideration of extension of the age of screening. 